\title{
ENERGY EFFICIENT CLUSTERING ALGORITHM FOR MOBILE CLUSTER HEADS TO ENHANCE THE LIFESPAN OF WIRELESS SENSOR NETWORK
}

\author{
Mr.S.Manikandan \\ Research Scholar, \\ Department of Computer Science, \\ AJK College of Arts and Science, Coimbatore \\ manicsphd@gmail.com \\ Dr.B.Suresh Kumar, \\ Assistant Professor, \\ Department of Computer Science, \\ AJK College of Arts and Science, Coimbatore. \\ sureshkumar@ajkcas.com
}

\begin{abstract}
Minimizing the formation of energy holes is an important research issues to be dealt in Wireless Sensor Networks. The contributions of the paper are two-fold. First we have proposed a novel energy efficient clustering algorithm along with a novel cluster head selection process which reduces the formation of energy holes in the network thus increasing the lifetime of wireless sensor network. Another significant contribution is that we have optimized the sojourn time of the cluster head based on the residual energy of the one hop sensors. We argue that our proposed work is efficient in dealing with various design and implementation issues of clustering procedures subsist in the existing works. The efficiency analysis is studied and presented. Also the time efficiency analysis of the proposed clustering algorithm is calculated. Simulation experiments showcase a noteworthy improvement in network lifetime in comparison with the existing algorithms.
\end{abstract}

Keywords: Wireless Sensor Network, WSN, clustering, cluster head, clustering algorithm, energy hole, bottle neck nodes and network lifetime.

\section{Introduction}

Wireless Sensor Network comprises of small tiny sensor nodes which are deployed in large numbers across the region to be monitored. Drastic improvements in the area of miniaturization led to the innovation of these small tiny sensor nodes which can be spread across large area with minimal cost. These sensor nodes have the ability of self-organizing themselves to form a wireless network. The low cost sensor nodes are deployed randomly in the area of interest usually in large numbers for the purpose of precise sensing. They are used to monitor various environmental conditions like humidity, moisture, pressure, temperature, sound, movement and so on. Wireless sensor networks offer a wide diversity of real time applications like environmental monitoring, crisis management ,military applications, medical applications, agricultural applications and so on[1]. One of the most important issues to be dealt with Wireless Sensor Network is the limited energy resources of the nodes [3]. Usually thousands of sensor nodes will be deployed in the sensing area, to monitor the physical conditions where human intervention is practically impossible. So recharging or replacement of the energy resources for each individual sensor node is extremely unfeasible. As a result it becomes crucial for the sensor nodes to minimize the energy consumption to a larger extent for an enhanced lifetime. Otherwise the nodes will exhaust their energy resources very fast and it highly minimize the lifetime of wireless sensor network.. Researchers have put their best efforts to frame algorithms and protocols which try to minimize the overall energy consumption and thus enhance the wireless sensor network lifetime. Another important issue to be dealt with sensor network is the formation of energy holes.[1,2] The nodes that are deployed in the monitoring area sense the required data and communicate them to the base station for data aggregation and decision making. All the nodes cannot communicate directly with the base station as the nodes are limited in their communication 
radius. Only those nodes where the base station is available within their communication radius can send the data packets directly to the base station. Other nodes have to pass the data packets through multihop communication. Due to this scenario nodes that are deployed near the base station will be overloaded and will lose their energy very soon as it becomes compulsory for them to forward packets in place of other sensor nodes in the network. Forwarding and receiving more number of packets tend to consume high amount of energy thus leading to their earlier death. So this in turn will have a huge impact on the lifetime of wireless sensor network. In order to minimize the hotspot formation around the base station, nodes are usually grouped together into clusters where each group will have a cluster head. Rather than communicating the data to the base station directly, the nodes will communicate their sensed data to the cluster head.[4] The cluster head will aggregate the sensed data and communicates it with the base station. This introduces a hierarchical structure in the network rather than a flat structure. There are so many short comings associated with flat network and there are so many advantages with clustering the network. The following are the drawbacks encountered with flat type of network [5].

- Sensor nodes will drain their energy very soon.

- Responsibilities like data collection and broadcasting of data will become tougher.

- Scalability becomes a tedious task.

- Flat network results in increasing the communication delay and network management complications.

The following are the advantages with clustering the network.

- In clustering the sensor nodes transmits the data to cluster heads rather than to the base station thus minimizing the overall energy consumption.

- Task like data collection, aggregation and dissemination will be handled by the cluster head rather than the individual node.

- Scalability of the network is supported

- A hierarchical structure is imposed which will ease the communication delay and network management complications.

- Enhances the lifetime of network by reducing the overall energy consumption of individual sensor nodes.

Flat network and Hierarchical network is depicted in Fig. 1. The advantages are clearly showcase in the diagram where the nodes in flat network communicates the data to base station through multi hop communication whereas nodes in hierarchical network are grouped into clusters and the sensed data is communicated to the cluster head rather than the base station.

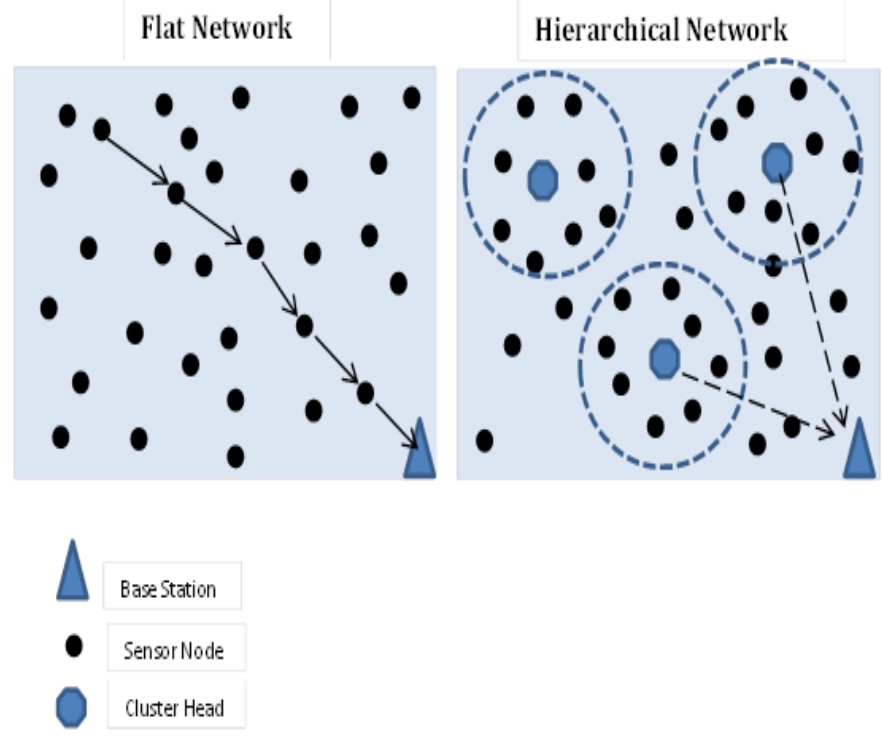

Fig. 1. Flat Network vs. Hierarchical Network

Cluster heads forms a second tier architecture in the sensor network. Cluster heads are termed as relay nodes of the network [5] and they are designated with the tasks of data collection, aggregation and transmission of data to the base station. Usually cluster heads are sensor nodes with high communication radius and rich in energy. The clustering algorithm proposed can be used to form clusters at different locations as required in the case of mobile cluster heads. Cluster heads are made mobile in order reduce the formation of hotspot in a specific region. Static cluster heads results in the formation of hotspots around them as similar to the one that occurred 
around the base station. To avoid this rather than keeping the cluster heads at fixed locations, mobile cluster heads are deployed in the network which changes its location frequently so as to avoid the formation of hotspots $[6,7]$.

In this paper we have presented a novel clustering algorithm which can be used for mobile cluster heads. The method of cluster head selection, the clustering algorithm to form clusters across the network is proposed. The paper if organized as follows. Section discusses the various related works in relevance to the proposed algorithm. Section 3 describes the proposed clustering algorithm with pseudo code. Section 4 describes the method used for data collection in the proposed algorithm. Section 5 discuss about the efficiency analysis of the clustering algorithm. This section analyzes the way in which the proposed clustering algorithm deals with the various design and implementation issues of clustering. The next section deals with the time efficiency analysis of the algorithm followed by the simulation experimental results and discussion.

\section{Related Work}

The pioneering work on clustering procedure is the work proposed in [1]. The authors formulated a clustering algorithm termed as Low Energy Adaptive Cluster Hierarchical (LEACH) routing protocol. In this clustering protocol, the energy backup of the cluster head is verified at the end of each data collection round in order to enhance the network lifetime. When the energy level of the cluster head falls below a specific threshold then a new cluster head selection process is initiated based on the probabilistic theory. The authors prove that the network lifetime is increased to a significant level since the election of cluster head is based on rotation basis.

Stable Election Protocol is another clustering algorithm introduced by the author in [2] where they categorized the nodes in to two types namely normal and advanced nodes. The Advanced nodes are defined as nodes with high residual energy and are the only nodes eligible for acting as cluster heads. Normal nodes do not possess the capabilities of becoming the cluster heads and they can be used just for forwarding the packets. The node with the highest residual energy becomes the cluster head and there is no need for collecting the residual energy of the entire network after the completion of every round.

This Hybrid Energy Efficient Distributed Protocol (HEED)[3] is designed with the intention of distributing a fair energy consumption across the entire network rather than on a localized areas. Localized energy consumption will decrease the network lifetime to a great extent. The HEED protocol operates on the three main aspects i.e. fair distribution of load across the entire network thereby increasing the network lifetime to a higher level, nondesignation of a node as cluster head after a specific number of rounds and finally the cluster heads are spread across the network in order to facilitate maximum number of nodes to access the cluster head.

The work discussed in [4] proposed another energy efficient routing protocol called Distributed Energy Efficient Hierarchical Clustering (DWEHC) Protocol where balanced equal sized clustered are created across the network for a fair load distribution. This also improvises the energy efficiency of the network and also enhances the intra cluster network topology. The cluster head selection is initiated after the sensors in the network computes its residual energy and a list of its one hop sensor nodes. After the first round the node with the highest residual energy among the one hop sensor nodes will be the next cluster head. A node which has the highest weight in the neighbourhood will be regarded as the $\mathrm{CH}$ and other nodes will become the member nodes.

The works in [8] deals the issue of hotspot formation around the sink node by changing the sink node position in Grid based clustering technique, which considers the optimal method for this purpose. The authors evaluated the clustering technique using MATLAB which shows a very good improvement in terms of network lifetime. The proposed algorithm minimizes the overall energy consumption which results in an increased throughput and high balance residual energy which obviously enhances the lifetime of network.

The authors of [9] propose an energy-efficient algorithm for routing in WSNs through an approach called density-based Fuzzy C-Means clustering (DFCM). This algorithm attains load balancing among the sensor nodes. Simulation experiments of this work exhibit a significantly good performance and the authors argue that the proposed algorithm is suitable for data collection in smart grids.

Parwekar et al [10] says that one of the best ways to reduce the energy consumption is to reduce the transmission distance between sensor nodes, cluster head and base station. He also states that Reducing the transmission distance is a NP-Hard problem. The author suggests utilizing the concept of optimization for solving this issue. The SGO optimization is said to minimize the transmission which in turns minimized the energy consumption of nodes for transmission. Experimental results evaluate the performance of SGO with Generic algorithm and Particle Swarm Optimization and it is concluded that SGO shows a very good performance in comparison with GA ad PSO. 


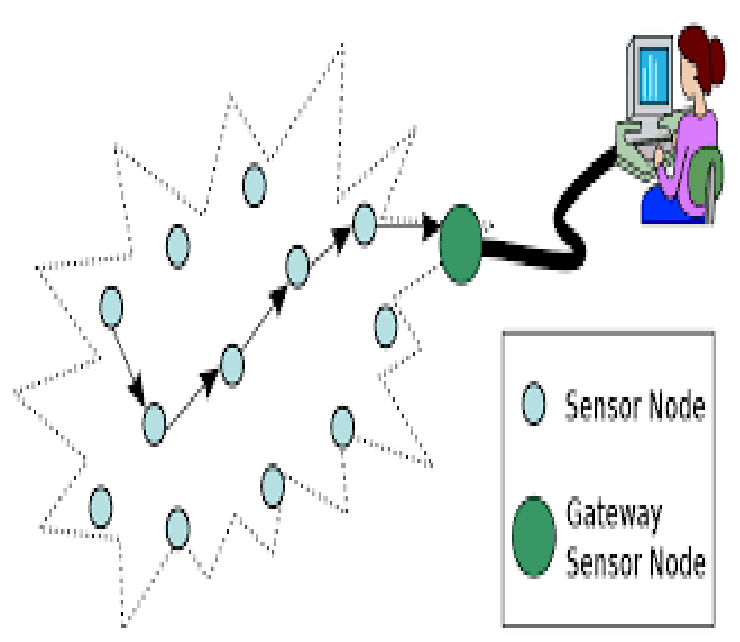

Fig. 2. Sensor Network Communications

Dao et al in [11] proposes a novel algorithm which identifies the reliability of the aggregated data in wireless sensor network. The process of sensing involves communicating the sensed data to the cluster head and aggregating them by the cluster heads and communicating the aggregated data to case station. Classification plays a significant part in this circumstance. The authors have suggested using Support Vector Machine classification and exploit the concept of improvised flower pollination to implement the critical parameters of Support vector machine (SVM). The authors argue that the improvised flower pollination algorithm Simulation experiments exhibits good performance of the proposed method in comparison with the existing algorithms.

Research work in [12] discuss about a novel clustering algorithm termed clustering algorithm (HQCA) which is supposed to produce clusters with good quality. The algorithm utilizes various quality parameters for measuring the features of clustering process and the authors argue that these parameters will produce good quality clusters in the network. The metric focus on parameters like inter cluster and intra cluster coordination, distance between clusters and error rate for improvising the cluster quality. Fuzzy logic is used to find the best cluster head considering parameters like residual energy, distance between the sensor nodes and cluster head, distance between the cluster head and base station. The proposed clustering algorithm provides various advantages on parameters like reliability, reduced rate of errors, scalability and performance in case of large networks. Experimental analysis indicates the proposed clustering algorithm increases the network lifetime to a great extent in comparison with the existing algorithms, Also the proposed algorithm minimizes the energy consumption and maximized the residual energy among the sensor nodes network lifetime.

The work proposed in [13] discusses on the concept of data aggregation and proposes a new aggregation technique based on the concept of extreme learning machine (ELM) and clustering process. The author argues that the proposed method is effective in reducing erroneous and redundant information. The paper deploys a distance-based radial basis function at the projection phase which is helpful to minimize the instability of the preparation phase. Also to filter the sensed data generated by each sensor node, Kalman filter is utilized. Experimental analysis showcases a significantly good performance of the proposed algorithm in terms of accuracy in clustering, energy efficiency over the existing clustering algorithms.

Research work [14] deploys the concept of clusters to increase the lifetime of wireless sensor network. The entire network is partitioned in to clusters using a three tier clustering algorithm. The author says the proposed clustering algorithm increase the network lifetime. The proposed algorithm categorizes the sensor nodes into three broad levels based on their residual energy of the nodes. Cluster head selection is done based on the proposed threshold value and energy parameters. The best cluster head selection helps in the improvising the performance of the entire network. Simulation experiments are carried out using MATLAB which showcase a better performance in terms of network lifetime in comparison with the existing methods.

The authors in work [15] propose a new fuzzy algorithm based clustering procedure which balances the load equally among the cluster heads which in turn has got a good impact on the performance. The proposed concept exploits the Ant colony optimization technique to identify the optimal routing path for transmitting the sensed data from the source node to the cluster head. Simulation experiments done to measure the performance of the proposed algorithm demonstrate that the proposed algorithm is effective and efficient in maintaining the residual energy in comparison with the existing algorithms. The proposed algorithm minimizes the total number of 
control messages and energy consumed in each node during data transmission which in turns enhances the network lifetime.

Paper [16] addresses the problem of lifetime maximization in WSNs by devising a novel clustering algorithm where clusters are formed dynamically. Specifically, the authors analyse the network lifetime maximization problem by balancing the energy consumption among cluster heads. Based on the analysis, they provide an optimal clustering technique, in which the cluster radius is computed using alternating direction method of multiplier. Next, the authors have proposed a novel On-demand, Optimal Clustering (OPTIC) algorithm for WSNs. The cluster head election procedure used in the paper adaptive which is dependent on the vitality of the occurrence of events. The authors argue that the proposed algorithm minimizes the message overheads and computations to a great extent. Simulation results show that the proposed algorithm improves the balance residual energy by $18 \%$ and the life span of the network is increased by a factor of $19 \%$ in comparison with the existing clustering protocols.

In paper [17], the authors have proposed a novel clustering technique which claims to be energy efficient based on the concept of genetic algorithm with a novel objective function. Here the objective functions take into consideration the following three parameters for the purpose of optimization.. Number of clusters, compactness and separation are the three factors taken for optimization. Simulation experiments showcase a significant improvement in the performance of the proposed algorithm in comparison with the algorithms like ERP, IHCR, and SEP

In the work proposed in [18] the authors consider a clustering algorithm which uses concepts based on probabilistic, adaptive, fuzzy logic, and Multi-attribute decision-making techniques for electing the cluster head. The authors argue that the proposed algorithm offer a better quality of service whereas the existing algorithms though provide a good improvement in network lifetime and other parameters but the quality of service is not good.

This work presented in [19] proposes a method called SPDAC (Shortest Path Discovery for Area Coverage) for extracting the shortest path for covering the network area. The proposed SPDAC algorithm aims to minimize the routing trajectory of the mobile relay nodes by using the concept of strategic deployment of nodes. Apart from this; the authors have also proposed another protocol as an extension which is a prediction-based clustering protocol for energy consumption (PCP-EC). The authors argue that the proposed algorithms enhance the life span of the network manifolds. Experiments are conducted by suing NS2 which exhibits the proposed algorithm shows a good performance in comparison with the existing algorithms,

\subsection{Issues involved in Clustering Process}

Many research papers in the past have experimented ample with forming energy efficient clustering algorithms which will enhance the lifetime of network. Forming energy efficient clustering algorithms has a direct impact on the enhancing the longevity of sensor network lifetime. There are so many important parameters to be considered while forming a clustering algorithm. The algorithm should see to that no overlapping clusters are formed. All the clusters should be of approximately the same size and amount of overhead on each cluster should be normalized. There are so many different design and implementation issues discussed in papers like[17]. The following are the various design and implementation issues to be dealt while framing an efficient clustering algorithm given in [17].
- Node Mobility
- Traffic load
- Overlapping clusters
- Load Balancing
- Dynamic Cluster Control
- Inter-Cluster Coordination
- Data Aggregation
- Fault tolerance
- Scalability
- Number of clusters
- Cluster Formation time
- Node heterogeneity
- Self-Configuration and Re- Configuration 
A good clustering algorithm should take into consideration the above said issues. Avoiding the above issues will make the clustering algorithm an efficient one.

\section{Contribution and Problem Definition of the Proposed Work}

\subsection{Contribution of the Paper}

The contributions of our research papers are as follows.

- The paper proposes a novel clustering algorithm for the wireless sensor network which efficiently deals with the various design and implementation issues of clustering.

- An optimal sojourn time for the mobile cluster head by proposing a linear programming formulation. The objective function maximizes the network lifetime by considering the residual energy of the one hop neighbors.

- A procedure to construct data collection trees for data collection by deploying Mobile Sinks.

- We also have a done analytical study on the efficiency of our clustering algorithm to deal with various critical design and implementation issues of clustering.

- $\quad$ Time efficiency analysis for the proposed clustering algorithm is also done.

\subsection{Problem Statement}

The problem statement is defined as follows:

Given a Wireless sensor network where the nodes are deployed for sensing their intended parameters. Construct a clustering algorithm for mobile cluster heads to form data collection trees at various sojourn spots. Also optimize the sojourn time of the cluster heads by framing a linear programming formulation based on the one hop residual energy to improvise the lifetime of the wireless sensor network.

The algorithm proposed employs a novel procedure to form data collection trees at various sojourn spots across the network. The algorithm is efficient in dealing with the various design and implementation issues of clustering in wireless sensor networks. Apart from the clustering procedure we also optimize the stopping time which in turn will optimally enhance the lifetime of wireless sensor network.

\section{Proposed Algorithm for Cluster Head Selection}

\subsection{Selection Procedure}

The proposed method initiates with the process of selecting the cluster head. At the time of deployment, nodes with high energy levels are selected with the intention of forming candidate nodes for cluster heads. Data collection is done in rounds of specific time duration. On the commencement of first round cluster heads are selected arbitrarily, as all the nodes possess an equal amount of initial energy. Once the cluster heads are selected, a clustering algorithm is initiated to decide on the cluster members. The following sets of steps are initiated to form data collection trees.

- The clustering algorithm starts by setting two parameters MyClusterID to 0 and MyHopCount to $\infty$.

- After this step the nodes designated as heads with high energy levels broadcasts a message incorporating its ID and HopCount where the HopCount is set to 1 .

- This broadcasted message will be received by the sensors that lie within the communication radius of the cluster head. The sensors that receive the message become the one hop sensors for the cluster head.

- The sensors that receive this message makes themselves member of the respective head and rebroadcast the same message by incrementing the HopCount.

- The next set of sensors which receives the message will become the second hop sensors and hence the HobCount will be 2 .

- The above process is repeated until the all the sensors become members of a particular cluster head.

- And there are also possibilities too far for one sensor to receive multiple messages from multiple cluster heads. In this scenario, the sensor compares the HopCount of the received messages.

- Then it joins with cluster head where the HoPCount is very minimal.

- In this way overlapping clusters are completely avoided, since a sensor will not be able to join two cluster heads according to the procedures in the algorithm. 


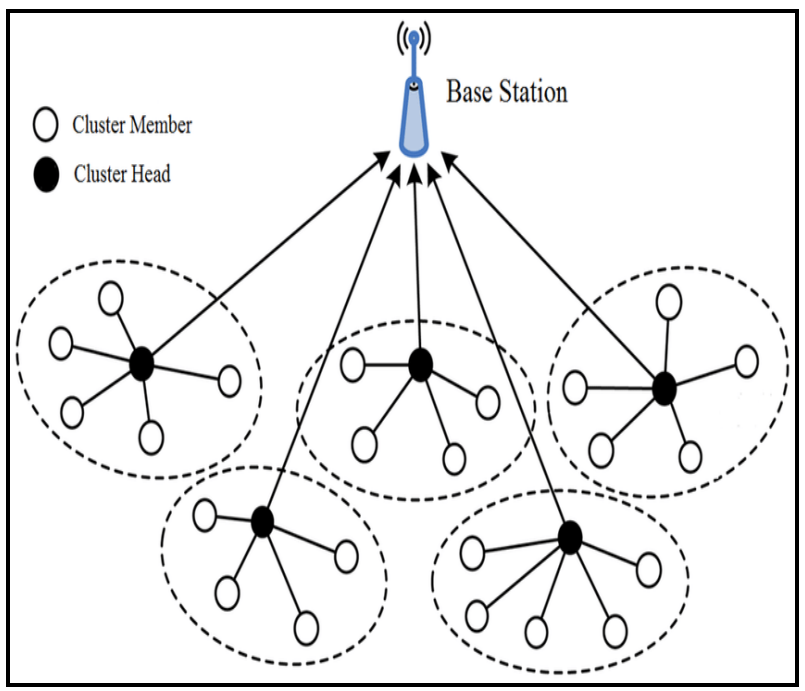

Fig. 3. Cluster Communication

To initiate the data collection process, all the cluster heads should initially call the proposed clustering algorithm. At the end of the clustering procedure, clustering trees will be formed across the network. These trees can then be used by the cluster heads or sinks to initiate the data collection. At the end of a particular round after the data collection process, the cluster heads in case if they are assumed to be mobile can be replaced to another location and the another set of clustering trees can be formed by calling the same procedure. The algorithm is efficient in terms of dealing with various implementation issues that occur during the process of clustering in a wireless network. We argue that our algorithm is efficient in handling the various implementation issues in an effective manner. The pseudo code for the proposed technique is represented below. The efficiency of our algorithm to deal with various design and implementation issues is discussed in the next section.

\subsection{Pseudo code for Proposed Clustering Algorithm}

\section{Procedure Cluster Formation( )}

\section{BEGIN}

1. Every sensor nodes sets two parameters

MyClusterID = 0

MyHopCount $=\infty$

2. Each Cluster head broadcast a (Cluster ID, HopCount $=1)$ message to the next level sensors.

3. Each sensors in the next level after receiving the message

4. Checks if HopCount is less than MyHopCount then sets the following BEGIN

MyClusterID = ClusterID;

MyHopCount -= HopCount

Next_Hop $=$ Sensor from which the message was received

HopCount $=$ HopCount +1

END

Broadcast the message (Cluster ID, HopCount ,) to the next level sensors

\section{ELSE}

END

Sensors ignore the message

\subsection{Network Partitioning and Data Collection Phase}

The area to be monitored is divided in to various equal size sectors. Each sector is assigned to a separate cluster head. The cluster head initiates the data collection process by starting at a random sojourn point at the periphery of the sector. A cluster initialization procedure is called to create data collection trees. Data collection phase is instigated and continues for an optimized time duration. At the expiry of the fixed time duration, the cluster head move three hops away from the current position. The sojourn spots are not predetermined or no enquiry for residual energy is made. Instead the cluster head moves three hops away from its current position as the fourth corona $\mathrm{z}$ sensor nodes will act as bottle neck nodes in the next data collection round. At the expiry of one round 
the cluster head moves to the inner sector to initiate the data collection phase. The network partitioning and the position of cluster head is depicted in Fig 4. The pathway for the cluster head to move in its sector for data collection is depicted in Fig.5

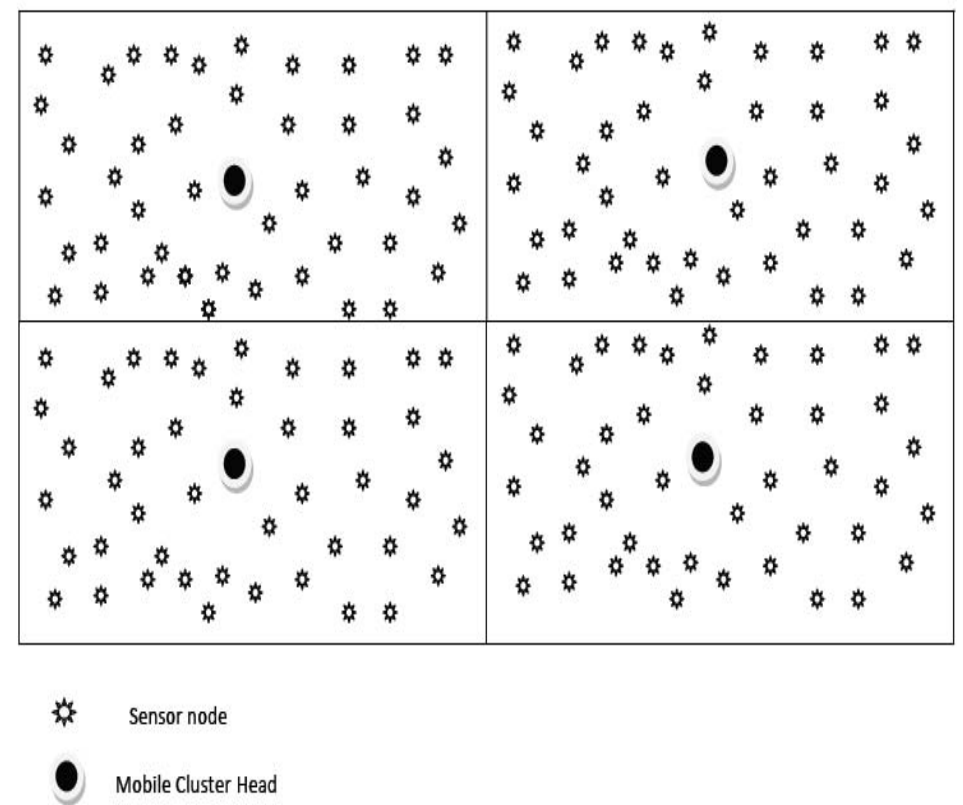

Fig. 4. Network Partitioning.

The network is divided into four sectors which require the deployment of four mobile cluster heads one in each sector. The number of cluster heads used in the network is proportional to the number of sector divisions.

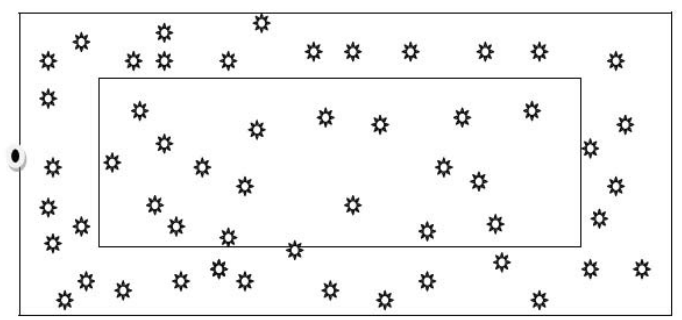

Fig. 5. Sector trajectory for Mobile Cluster Head

The trajectory is designed in such a way that the sensors in fourth corona will acts as the bottle neck nodes for the next data collection phase. It is assumed that the fourth corona sensors would have consumed less energy in comparison with the first, second and third corona nodes. Those nodes have consumed more energy as they forward data packets on behalf of nodes at the outer coronas. So the trajectory is designed in such a way that it tries to eliminate overloaded nodes in the previous data collection phase to act as the bottle neck nodes for the current round.

\subsection{Optimization of Stopping Time}

The sojourn time of the cluster head is optimized by means of linear programming formulation proposed below. Rather than making the cluster head to stop for a fixed time period for the data collection round, the stopping time is optimized for a amount of time which will lead to optimal network lifetime. The objective function of the linear programming formulation is to maximize the network lifetime which is defined as summation of the sojourn time of the mobile cluster heads at various topping locations. The constraints are defined to check that 
the one hop residual energy should be less than a threshold value defined and the stopping time of the cluster head should always be greater than zero.

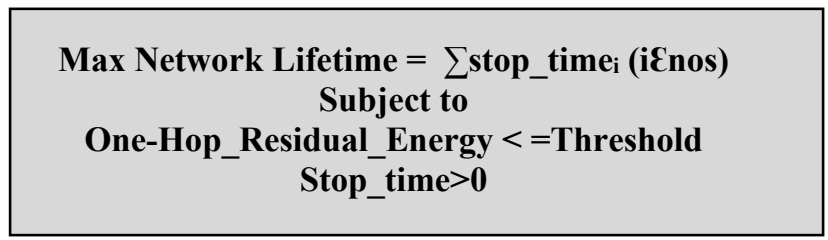

\section{Efficiency Analysis of the Proposed Clustering Algorithm}

The section explains on the various design and implementation issues the clustering algorithm can effectively handle.

\section{Overlapping Clustering}

Overlapping cluster is a term used to refer the members of one clusters may become the members of another cluster. In other words a particular sensor node can be a member node in two or more clusters. This issues has to dealt effectively as it will cause issues during the data gathering process. Our proposed algorithm guarantees that one senor node will be a member of only one cluster as each sensor nodes avoids the messages received from other cluster heads by comparing the hop count.

\section{Load Balancing}

Load balancing refers to equal distribution of loads among all the cluster heads. The proposed clustering algorithm forms equal sized clusters across the network, so the load will obviously be balanced without overloading a specific set of sensor nodes. Overloading a specific set of sensor nodes leads to formation of energy holes in the network which in turn will decrease the life span of the network to a great extent.

\section{Scalability}

Scalability refers to the ability of the clustering algorithm to add or delete member nodes in the cluster. The proposed clustering algorithm is efficient in node addition or deletion from the cluster. When a node is added, according to the procedure the node will join the cluster head with minimal hop count from the particular sensor nodes. Similarly the algorithm is also efficient in removal of nodes or dealing with expired nodes. The link of the expired nodes in multi hop communication will be replaced with another link in case of such failures.

\section{Cluster Formation time}

The amount of time taken by the clustering algorithm to form clusters should be very minimal as it will affect the data collection process. The time complexity of the algorithm is analyzed in the following section for the proposed algorithm.

\section{Self-Configuration and Re-configuration}

This represents the amount of time taken by the algorithm to self-configure itself when required. Our proposed algorithm has the capability to reconfigure very fast when needed. This helps in minimizing the amount of time taken for forming new cluster groups. At any point of time during the data gathering operation the cluster can be configured as well as reconfigured at a minimal amount of time.

\section{Dealing with Mobility}

The proposed clustering algorithm can deal with node mobility in an efficient manner. A mobile node which changes its location can automatically get connected to the cluster head with minimal hop count. But the cluster should reconfigure at regular intervals to deal with the mobility of the nodes.

\section{Data Aggregation}

Data aggregation in the proposed algorithm is done by using multi-hop communication. The clustering tree formed will aggregate the data by passing the sensed data to its immediate neighbor. The message reaches the cluster head by means of multi hop communication. 
These various design and implementation issues which become very tedious for a clustering algorithm to deal are efficiently handled by the proposed clustering algorithm. The proposed clustering algorithm can be used by the mobile cluster heads to reduce the formation of hotspot around the cluster head thus increasing the lifetime of sensor network.

\subsection{Time Efficiency Analysis of the Clustering Algorithm}

The time efficiency of the algorithm refers to the amount of time take by the algorithm to complete its intended task. The analysis of the algorithm is done as follows.

\section{Analysis}

- The basic operation of the proposed algorithm is Comparison. The sensor nodes perform comparison operation a number of times to find the cluster head with minimal hop count. The sensor node will become the member to the cluster head with minimal count.

- The next process in the analysis to find the basic operation count. The number of times the basic operation is carried out depends on the total number of sensor nodes in the network and the total number of cluster heads.

- Let us assume there are " $\mathrm{N}$ " numbers of sensor nodes and " $\mathrm{X}$ " number of cluster heads.

- Each sensor node will compare the hop count received from each cluster head with MyHopCount which is initially initialized to $\infty$. If the received hop count is less than its MyHopCount then the new hop count will be assigned to MyhopCount. This process is repeated by all the senor nodes

- Let the number of messages received by a particular sensor node for checking the hop count be "M"

- The amount of comparison operation done by the sensor node will be NXM as the sensor node has to compare all the hop count messages received with MyHopcount to finalize the hopcount.

- So, the running time of the proposed algorithm is fixed as $\mathrm{O}(\mathrm{NM})$ where $\mathrm{N}$ represents the number of sensor nodes in the network and $\mathrm{M}$ represent the amount of messages received by each sensor node.

\section{Results and Discussions}

The efficiency of the proposed algorithm is compared with various existing algorithms where the cluster head is placed at various random locations such as at the center of network, periphery of the network and multiple cluster heads. Simulations parameter for experiments are following; BS is Cluster head is deployed in a heterogeneous network $\mathrm{N}=100100 \mathrm{~m} \times 100 \mathrm{~m}$ field size. The existing algorithm is compared with conventional static sink category and various other existing categories like random cluster heads, mobile cluster heads at the periphery of the network. The proposed algorithm showcases a very good improvement over other categories.

The following table represents the simulation parameters used in the experimental analysis

$$
\text { TABLE I SIMULATION PARAMETERS }
$$

Network Dimensions 100m *100m

Number of Nodes 100

Initial Energy .5 joules 


\begin{tabular}{|l|}
\hline Percentage of CHs .1 \\
\hline Data Aggregation $50 \mathrm{pj} / \mathrm{bit} \mathrm{j}$ \\
\hline Packet size $4000 \mathrm{bit}$ \\
\hline Transmitter Electronics $50 \mathrm{nj} / \mathrm{bit}$ \\
\hline Receiver Electronics $50 \mathrm{nj} / \mathrm{bit}$ \\
\hline Transmit amplifier $100 \mathrm{pj} / \mathrm{bit} / \mathrm{m} 2$ \\
\hline
\end{tabular}

Four types of cluster head placement is experimented i)static cluster head, ii) multiple cluster heads randomly deployed in the network, iii) cluster head deployed at the periphery of network and iv)cluster head deployed according to the proposed algorithm . The lifetime of the network for all the four categories are identified. The following graph depicts the lifetime of sensor network for all the four categories (Fig. 4). The proposed clustering algorithm shows a significant improvement in terms of network lifetime over other three categories

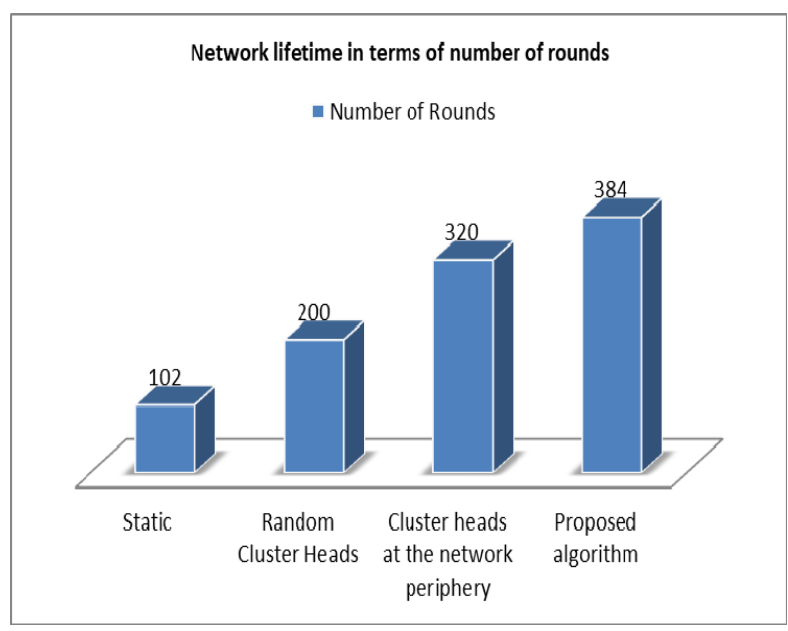

Fig. 6. Network Lifetime (till the death of one sensor node)

. The network lifetime is categorized to be measure in three ways.

- Network lifetime till the expiry of one node

- Network lifetime until the expiry of $5 \%$ of sensor nodes

- Network lifetime until the expiry of $10 \%$ of sensor nodes

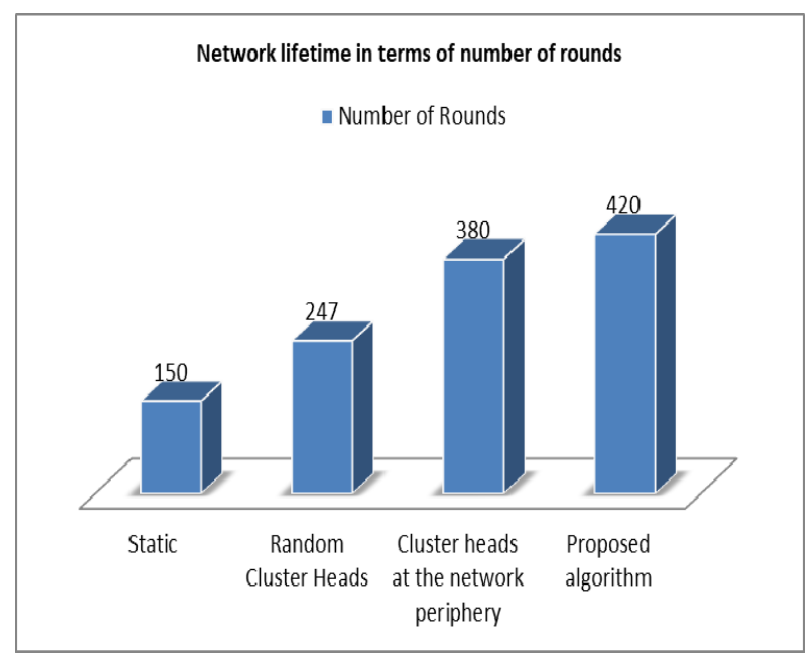

Fig. 7. Network Lifetime (till the death of 5\%of sensor nodes) 
In all the three cases the proposed clustering algorithm showcases a significant improvement over the other three catgories. The results are depicted in three graphs(Fig. 4,5, and 6).

Likewise the number of alive nodes after a sepcific set of rounds are observed. This experiments also proves the proposed algorithm has got more number of alive nodes when comapred to the other three catgories. The graph is depicted in Fig. 7. The graph indicates there are more number of live nodes in the proposed algorithm in comparison with static clsuter head, cluster head at the periphery and random cluster heads methods. Number of live nodes plays an important role in lifetime enhancement of sensor network.

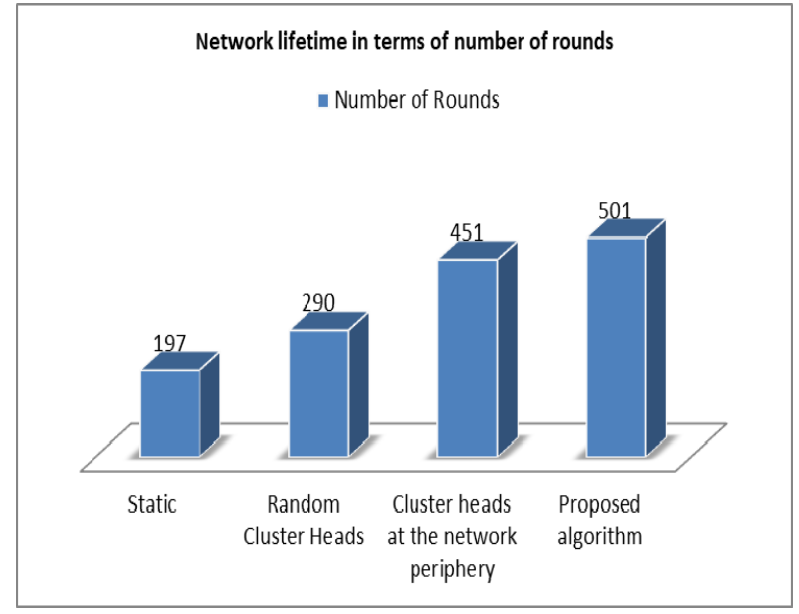

Fig. 8. Network Lifetime (till the death of $10 \%$ of sensor nodes)

Likewise the number of dead nodes are also measured after a certain number of rounds.(Fig. 7) The results indicates that there are less number of dead nodes in the proposed algorithm when compared to the other categories. Similarly the number of alive nodes are also very high in the proposed scheme when compared to the other algorithms.

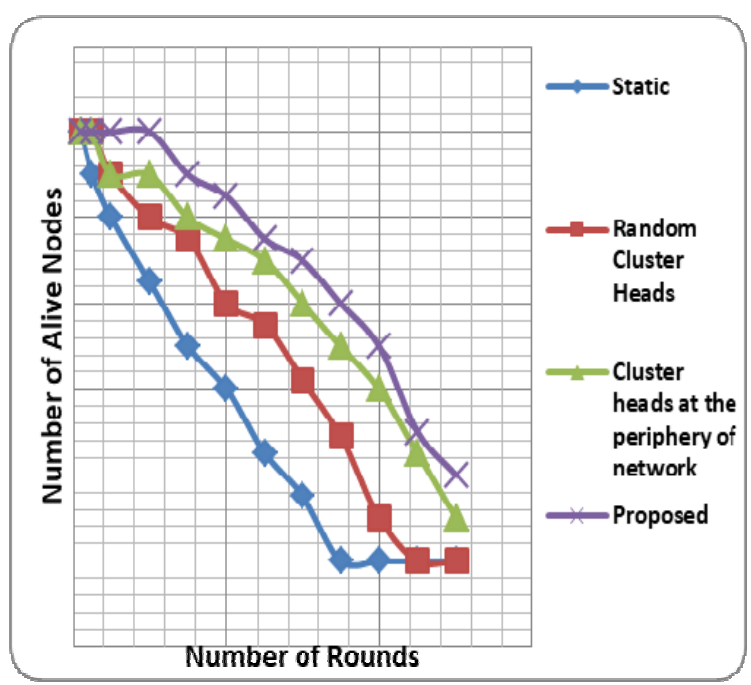

Fig. 9. Number of Alive nodes

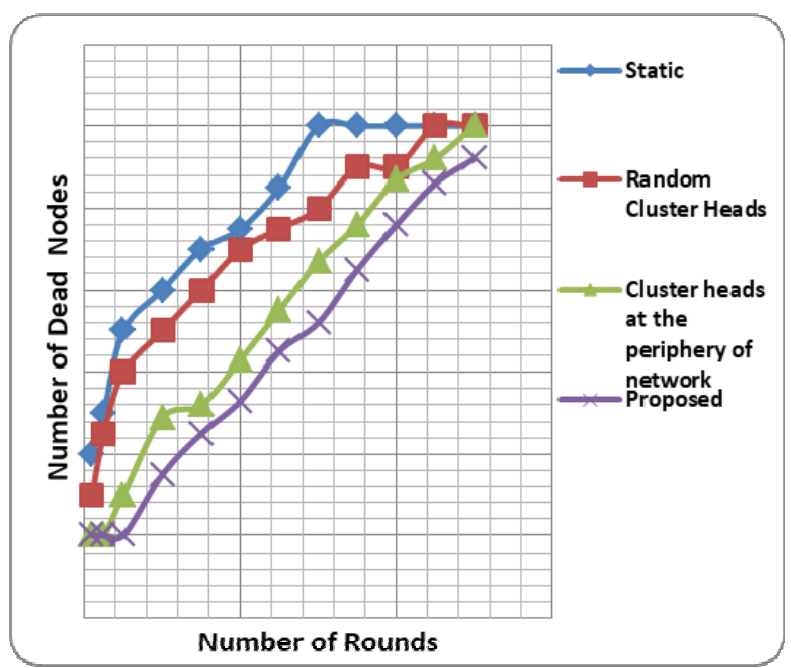

Fig. 10. Number of Dead nodes

\section{Conclusion and Future Work}

The paper proposes a sector based division of the sensor network and deployment of mobile cluster heads in each zone for data collection. A new clustering algorithm is proposed to form data collection treeThe . The proposed clustering algorithm efficiently deals with various design and implementation issues involved in clustering process. Also we have proposed a data collection method by using the clustering tree formed by using a mobile sink. The efficiency of the clustering algorithm to deal with various implementation issues is also discussed. Running time of the algorithm is calculated by doing a time efficiency analysis. Simulation experiments show a very good improvement of the proposed algorithm over the other categories of clustering algorithms. 
Our future work will involve framing an autonomous moving strategy for cluster heads to collect across the network. The autonomous moving strategy will try to find energy rich zones and stops at that location for an optimal amount of time to increase the overall network lifetime.

\section{References}

[1] W. Rabiner Heinzelman and H. Balakrishnan, 2000. Energy-Efficient communication Protocol for Wireless microsensor networks, IEEE, Proceeding of the 3rd Hawali International Conference on System Science.

[2] W. Heinzelman, A. Chandrakasan, and H. Balakrishnan, 2002. An application specific protocol architecture for wireless microsensor networks, IEEE Transactions on Wireless Communications.

[3] I.F. Akyildiz et al., Wireless sensor networks: a survey, Computer Network.

[4] Georgios Smaragdakis and Ibrahim Matta, 2004. SEP: A Stable Election Protocol for Clustered

[5] Pradeepa, K., W. Regis Anne, and S. Duraisamy. "Design and implementation issues of clustering in wireless sensor networks." International Journal of Computer Applications 47.11 (2012).

[6] Pradeepa, K., W. Regis Anne, and S. Duraisamy. "Improved sensor network lifetime using multiple mobile sinks: A new predetermined

[7] trajectory." 2010 Second International conference on Computing, Communication and Networking Technologies. IEEE, 2010.

[8] Pradeepa, K., and S. Duraisamy. "Energy efficient positioning of mobile base stations to improve wireless sensor network lifetime." International Journal of Sensor Networks 20.2 (2016): 92-103.

[9] Kamil, Aya Ahkam, Maham Kamil Naji, and Hasan Abdulhadi Turki. "Design and implementation of grid based clustering in WSN using dynamic sink node." Bulletin of Electrical Engineering and Informatics 9.5 (2020): 2055-2064.

[10] Kalaimani, Deepa, Zaheeruddin Zah, and Shruti Vashist. "Energy-efficient density-based Fuzzy C-means clustering in WSN for smart grids." Australian Journal of Multi-Disciplinary Engineering (2020): 1-16.

[11] Parwekar, Pritee. "SGO a new approach for energy efficient clustering in WSN." Sensor Technology: Concepts, Methodologies, Tools, and Applications. IGI Global, 2020. 716-734.

[12] Dao, Thi-Kien, et al. "Identification failure data for cluster heads aggregation in WSN based on improving classification of SVM." IEEE Access 8 (2020): 61070-61084.

[13] Baradaran, Amir Abbas, and Keivan Navi. "HQCA-WSN: High-quality clustering algorithm and optimal cluster head selection using fuzzy logic in wireless sensor networks." Fuzzy Sets and Systems 389 (2020): 114-144.

[14] Ullah, Ihsan, and Hee Yong Youn. "Efficient data aggregation with node clustering and extreme learning machine for WSN." The Journal of Supercomputing (2020): 1-27.

[15] Priyadarshi, Rahul, et al. "Three level heterogeneous clustering protocol for wireless sensor network." Microsystem Technologies (2020): 1-10.

[16] Bhushan, Bharat, and Gadadhar Sahoo. "ISFC-BLS (intelligent and secured fuzzy clustering algorithm using balanced load sub-cluster formation) in WSN environment." Wireless Personal Communications 111.3 (2020): 1667-1694.

[17] Ghosal, Amrita, Subir Halder, and Sajal K. Das. "Distributed on-demand clustering algorithm for lifetime optimization in wireless sensor networks." Journal of Parallel and Distributed Computing 141 (2020): 129-142.

[18] Pal, Raju, Subash Yadav, and Rishabh Karnwal. "EEWC: energy-efficient weighted clustering method based on genetic algorithm for HWSNs." Complex \& Intelligent Systems (2020): 1-10.

[19] Gupta, Rajeev. "Cluster Head Election in Wireless Sensor Network: A Comprehensive Study and Future Directions.", International Journal of Computer Networks and Applications

[20] Abhilash, C. N., et al. "Shortest Path Discovery for Area Coverage (SPDAC) Using Prediction-Based Clustering in WSN." Advances in Artificial Intelligence and Data Engineering. Springer, Singapore, 2021. 1345-1357.

\section{Authors Profile}

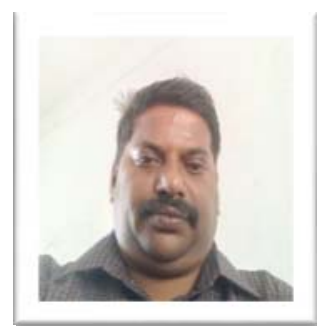

Dr.B.Suresh Kumar has completed his Ph.D in Computer Science in 2016 at Karpagam University, Coimbatore. He has obtained M.phil in Computer Science from Manomaniam Sundaranar University in 2003 and M.Sc in Computer Science from Thanthai Hans Roever College, Bharathidasan University. He has 21 years of academic experience and published more than 15 papers in reputed International journals and International conferences. $\mathrm{He}$ is currently working as Assistant professor at AJK College of Arts and Science Coimbatore. His areas of interest include Wireless Senor Network and Big Data Analytics.

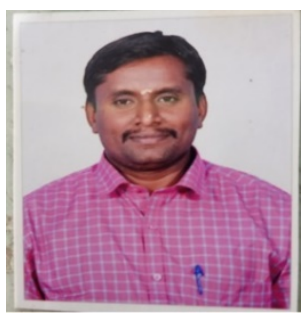

Mr.S.Manikandan has completed his UG in Computer Science from Bharathiar University and Post -Graduate in Computer Nandha Engineering College Erode. He has completed his Master of Philosophy under Bharathiar University and currently pursuing his research in Wireless Sensor Networks at AJK College of Arts and Science under Bharathiar University. He has published many papers in National and International Journals and he got a decade of experience in teaching core papers of Computer Science. His area of interest include Wireless Sensor Networks and Mobile Ad-Hoc Networks. 